2

3

4

5

$6 \quad{ }^{[1]}$ Department of Chemical Engineering, University of Patras, Patras, Greece

8

9

10

11

12

13

14

15

16

17

18

19

20

21

\section{concentrations in Europe}

David Patoulias $^{1,2}$ and S. N. Pandis ${ }^{1,2}$
${ }^{[2]}$ Institute of Chemical Engineering Sciences, Foundation for Research and Technology - Hellas

(FORTH/ICE-HT), Patras, Greece

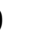

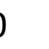

1

12

3

4

15

6

7

8

9

0

1


Table S1: Summary of parameters for each simulation

\begin{tabular}{|c|c|c|c|}
\hline CASE & Anthropogenic & ELVOCs & IVOCs \\
\hline $\begin{array}{c}1 \\
\text { (Base case) }\end{array}$ & \multirow{3}{*}{$\begin{array}{c}\text { Aging with } \mathrm{OH} \\
\mathrm{k}=10^{-11} \mathrm{~cm}^{3} \mathrm{molec}^{-1} \mathrm{~s}^{-1}\end{array}$} & $\begin{array}{c}\text { Monoterpene } \\
\text { oxidation 5\% } \\
\text { molar yield }\end{array}$ & $\begin{array}{c}\text { Yes } \\
\text { Aging reaction: } \mathrm{k}=4 \times 10^{-11} \mathrm{~cm}^{3} \mathrm{molec}^{-1} \mathrm{~s}^{-1}\end{array}$ \\
\hline 2 & & No & $\begin{array}{c}\text { Yes } \\
\text { Aging reaction: } \mathrm{k}=4 \times 10^{-11} \mathrm{~cm}^{3} \mathrm{molec}^{-1} \mathrm{~s}^{-1}\end{array}$ \\
\hline 3 & & $\begin{array}{c}\text { Monoterpene } \\
\text { oxidation 5\% } \\
\text { molar yield }\end{array}$ & No \\
\hline
\end{tabular}

23

24

25

26

27

28

Table S2: Boundary and initial conditions of the gases, PM, and number concentration of the simulation.

\begin{tabular}{|c|c|c|c|c|c|}
\hline & \multicolumn{4}{|c|}{ Boundary conditions } & \multirow[b]{2}{*}{ Initial conditions } \\
\hline Gas (ppb) & WEST & EAST & SOUTH & NORTH & \\
\hline Sulfur dioxide $\left(\mathrm{SO}_{2}\right)$ & $1 \times 10^{-2}$ & $1 \times 10^{-2}$ & $1 \times 10^{-2}$ & $1 \times 10^{-2}$ & $1 \times 10^{-2}$ \\
\hline Ozone $\left(\mathrm{O}_{3}\right)$ & 35 & 35 & 35 & 35 & 35 \\
\hline Nitric acid $\left(\mathrm{HNO}_{3}\right)$ & 0.1 & 0.1 & 0.1 & 0.1 & 0.1 \\
\hline Formaldehyde (FORM) & $5 \times 10^{-2}$ & $5 \times 10^{-2}$ & $5 \times 10^{-2}$ & $5 \times 10^{-2}$ & $5 \times 10^{-2}$ \\
\hline Ammonia $\left(\mathrm{NH}_{3}\right)$ & $5 \times 10^{-2}$ & $5 \times 10^{-2}$ & $5 \times 10^{-2}$ & $5 \times 10^{-2}$ & $5 \times 10^{-2}$ \\
\hline Ethene (ETH) & $5 \times 10^{-3}$ & $5 \times 10^{-3}$ & $5 \times 10^{-3}$ & $5 \times 10^{-3}$ & $5 \times 10^{-3}$ \\
\hline Carbon monoxide (CO) & 50 & 50 & 50 & 50 & 50 \\
\hline $\begin{array}{l}\text { Peroxynitric acid } \\
\left(\mathrm{HNO}_{4}\right)\end{array}$ & $1 \times 10^{-6}$ & $1 \times 10^{-6}$ & $1 \times 10^{-6}$ & $1 \times 10^{-6}$ & $1 \times 10^{-6}$ \\
\hline Aerosol $\left(\mu \mathrm{g} \mathrm{m}^{-3}\right)$ & & & & & \\
\hline Dust $(2.5 \mu \mathrm{m}-10 \mu \mathrm{m})$ & $1 \times 10^{-9}$ & $1 \times 10^{-9}$ & $1 \times 10^{-9}$ & $1 \times 10^{-9}$ & $1 \times 10^{-9}$ \\
\hline Sulfate (40 nm - $2.5 \mu \mathrm{m})$ & $1 \times 10^{-9}$ & $1 \times 10^{-9}$ & $1 \times 10^{-9}$ & $1 \times 10^{-9}$ & $1 \times 10^{-9}$ \\
\hline $\operatorname{SOA}(40 \mathrm{~nm}-2.5 \mu \mathrm{m})$ & $6.6 \times 10^{-7}$ & $6.6 \times 10^{-7}$ & $6.6 \times 10^{-7}$ & $6.6 \times 10^{-7}$ & $6.6 \times 10^{-7}$ \\
\hline Number conc. $\left(\mathrm{cm}^{-3}\right)$ & 50 & 50 & 50 & 50 & 50 \\
\hline
\end{tabular}


Table S3: Description of atmospheric measurement sites in Europe used in this work.

\begin{tabular}{|c|c|c|c|c|}
\hline Station & Name & Country & Longitude & Latitude \\
\hline ANB & Annaberg-Buchholz & Germany & 50.5717 & 12.9989 \\
\hline ASP & Aspvreten & Sweden & 58.8000 & 17.3833 \\
\hline BRK & Birkenes II & Norway & 58.3885 & 8.2520 \\
\hline $\mathrm{CBW}$ & Cabauw Zijdeweg & Netherlands & 51.9703 & 4.9264 \\
\hline DSN & Dresden-Nord & Germany & 51.0650 & 13.7414 \\
\hline DSW & $\begin{array}{c}\text { Dresden- } \\
\text { Winckelmannstrasse }\end{array}$ & Germany & 51.0361 & 13.7306 \\
\hline FNK & Finokalia & Greece & 35.3167 & 25.6667 \\
\hline GDN & Giordan Lighthouse & Malta & 36.0722 & 14.2184 \\
\hline $\mathrm{HOH}$ & Hohenpeissenberg & Germany & 47.8015 & 11.0096 \\
\hline HYY & Hyytiala & Finland & 61.8500 & 24.2833 \\
\hline ISP & Ispra & Italy & 45.8000 & 8.6333 \\
\hline KPU & K-puszta & Hungary & 46.9667 & 19.5833 \\
\hline $\mathrm{KST}$ & Kosetice (NOAK) & $\begin{array}{c}\text { Czech } \\
\text { Republic }\end{array}$ & 49.5734 & 15.0803 \\
\hline MLP & Melpitz & Germany & 51.5301 & 12.9339 \\
\hline MNT & Montseny & Spain & 41.7667 & 2.3500 \\
\hline PRG & Prague-Suchdol & $\begin{array}{c}\text { Czech } \\
\text { Republic }\end{array}$ & 50.1264 & 14.3846 \\
\hline USM & Usti n.L.-mesto & $\begin{array}{c}\text { Czech } \\
\text { Republic }\end{array}$ & 50.6611 & 14.0403 \\
\hline VAV & Vavihill & Sweden & 56.0167 & 13.1500 \\
\hline VRR & Varrio & Finland & 67.7667 & 29.5833 \\
\hline VSM & TMNT09 Vielsalm & Belgium & 50.3040 & 6.0013 \\
\hline WLD & Waldhof & Germany & 52.8022 & 10.7594 \\
\hline ZUG & $\begin{array}{c}\text { Zugspitze- } \\
\text { Schneefernerhaus }\end{array}$ & Germany & 47.4165 & 10.9796 \\
\hline NEO & Costa Navarino & Greece & 36.9932 & 21.6572 \\
\hline PAT & Patra-ICE-HT & Greece & 38.2980 & 21.8092 \\
\hline $\mathrm{SPC}$ & San Pietro Capofiume & Italy & 44.6553 & 11.6236 \\
\hline THE & Thessaloniki & Greece & 40.6166 & 23.0333 \\
\hline
\end{tabular}


Table S4a: AMS $\mathrm{PM}_{1}$ composition atmospheric measurement sites.

\begin{tabular}{|c|c|c|c|c|c|}
\hline Station & Name & Country & Longitude & Latitude & Altitude (m) \\
\hline FIN & Finokalia & Greece & 35.3167 & 25.6667 & 250 \\
\hline PAT & Patra & Greece & 38.2980 & 21.8092 & 85 \\
\hline BOL & Bologna & Italy & 44.4833 & 11.3333 & 0 \\
\hline SPC & San Pietro Capofiume & Italy & 44.6553 & 11.6236 & 11 \\
\hline
\end{tabular}

Table S4b: Filter-based $\mathrm{PM}_{2.5}$ composition measurements sites.

\begin{tabular}{|c|c|c|c|c|c|}
\hline Station & Name & Country & Longitude & Latitude & Altitude (m) \\
\hline CH02 & Payerne & Switzerland & 46.8131 & 6.9447 & 489 \\
\hline DE44 & Melpitz & Germany & 51.5301 & 12.9339 & 86 \\
\hline ES1778 & Montseny & Spain & 41.7667 & 2.3500 & 700 \\
\hline IT04 & Ispra & Italy & 45.8000 & 8.6333 & 209 \\
\hline PL05 & Diabla Gora & Poland & 54.1500 & 22.0667 & 157 \\
\hline SI08 & Iskrba & Slovenia & 45.5667 & 14.8667 & 520 \\
\hline
\end{tabular}

42

43

44

45

46

47

48

49

50

51

52

53

54

55

56

57 
60

\begin{tabular}{|c|c|c|c|c|c|c|c|c|}
\hline Station & $\begin{array}{c}\text { Mean } \\
\text { Observed }\end{array}$ & $\begin{array}{c}\text { Mean } \\
\text { Predicted }\end{array}$ & NMB & NME & $\begin{array}{c}\text { Mean } \\
\text { Observed }\end{array}$ & $\begin{array}{c}\text { Mean } \\
\text { Predicted }\end{array}$ & NMB & NME \\
\hline & $\left(\mathrm{cm}^{-3}\right)$ & $\left(\mathrm{cm}^{-3}\right)$ & $(\%)$ & $(\%)$ & $\left(\mathrm{cm}^{-3}\right)$ & $\left(\mathrm{cm}^{-3}\right)$ & $(\%)$ & $(\%)$ \\
\hline & \multicolumn{4}{|c|}{$\mathbf{N}_{10}$} & \multicolumn{4}{|c|}{$\mathbf{N}_{100}$} \\
\hline ANB & 8057 & 6763 & -16 & 37 & 1518 & 944 & -38 & 46 \\
\hline ASP & 2130 & 5310 & 149 & 149 & 552 & 748 & 36 & 57 \\
\hline BRK & 1878 & 3158 & 68 & 89 & 607 & 403 & -34 & 64 \\
\hline CBW & 13101 & 10223 & -22 & 29 & 1627 & 1448 & -11 & 16 \\
\hline DSN & 10591 & 6727 & -36 & 39 & 1976 & 1144 & -42 & 45 \\
\hline DSW & 7706 & 6364 & -17 & 38 & 1426 & 1123 & -21 & 34 \\
\hline FNK & 3962 & 5561 & 40 & 42 & 1760 & 2275 & 29 & 35 \\
\hline GDN & 5712 & 6805 & 19 & 33 & 2492 & 2695 & 8 & 28 \\
\hline $\mathrm{HOH}$ & 3438 & 3116 & -9 & 40 & 1011 & 630 & -38 & 40 \\
\hline HYY & 2207 & 2488 & 13 & 29 & 677 & 566 & -16 & 28 \\
\hline ISP & 6232 & 6636 & 6 & 45 & 1775 & 1227 & -31 & 39 \\
\hline KPU & 5269 & 6069 & 15 & 45 & 1543 & 1797 & 16 & 26 \\
\hline KST & 3596 & 5047 & 40 & 51 & 1123 & 1100 & -2 & 25 \\
\hline MLP & 5583 & 6223 & 11 & 43 & 1214 & 1064 & -12 & 28 \\
\hline MNT & 6455 & 8736 & 35 & 50 & 1492 & 1680 & 13 & 44 \\
\hline PRG & 7272 & 7586 & 4 & 46 & 1177 & 1224 & 4 & 27 \\
\hline USM & 15171 & 8764 & -42 & 51 & 1657 & 1050 & -37 & 40 \\
\hline VAV & 3250 & 8496 & 161 & 161 & 766 & 922 & 20 & 49 \\
\hline VRR & 1107 & 1107 & 0 & 51 & 324 & 158 & -51 & 63 \\
\hline VSM & 2903 & 7504 & 158 & 158 & 704 & 729 & 3 & 32 \\
\hline WLD & 4956 & 8079 & 63 & 69 & 1116 & 993 & -11 & 21 \\
\hline ZUG & 1237 & 2450 & 98 & 114 & 555 & 497 & -11 & 36 \\
\hline NEO & 2864 & 5179 & 81 & 83 & 1489 & 1930 & 30 & 41 \\
\hline PAT & 4705 & 5305 & 13 & 47 & 1747 & 1754 & 0 & 23 \\
\hline SPC & 8301 & 7451 & -10 & 35 & 1702 & 2020 & 19 & 35 \\
\hline THE & 3894 & 8830 & 127 & 127 & 1387 & 2347 & 69 & 72 \\
\hline ALL & 4820 & 6125 & 27 & 65 & 1160 & 1240 & 5 & 43 \\
\hline
\end{tabular}

Table S5: Prediction skill metrics of PMCAMx-UF for the case without IVOCs against daily ground measurements of particle number concentration above $10 \mathrm{~nm}\left(N_{10}\right)$ and $100 \mathrm{~nm}\left(N_{100}\right)$ during 5 June -8 July 2012.
61

2




\begin{tabular}{|c|c|c|c|c|c|}
\hline Station & $\begin{array}{c}\text { Mean } \\
\text { Predicted } \\
\left(\mu \mathrm{g} \mathrm{m}^{-3}\right)\end{array}$ & $\begin{array}{c}\text { Mean } \\
\text { Observed } \\
\left(\mu \mathrm{g} \mathrm{m}^{-3}\right)\end{array}$ & $\begin{array}{c}\text { NMB } \\
(\%)\end{array}$ & $\begin{array}{l}\text { NME } \\
(\%)\end{array}$ & $\begin{array}{c}\text { Factor of } 2 \\
(\%)\end{array}$ \\
\hline FIN & 2.98 & 2.12 & 40 & 44 & 87 \\
\hline PAT & 2.45 & 3.80 & -35 & 35 & 90 \\
\hline BOL & 4.29 & 5.68 & -24 & 35 & 74 \\
\hline SPC & 4.32 & 3.98 & 9 & 40 & 83 \\
\hline ALL & 3.56 & 3.79 & -6 & 38 & 83 \\
\hline
\end{tabular}

65

66

67 68

\begin{tabular}{|c|c|c|c|c|c|c|c|}
\hline \multirow{2}{*}{ Name } & \multirow[t]{2}{*}{ Station } & \multirow[t]{2}{*}{ Country } & \multirow{2}{*}{$\begin{array}{c}\text { Mean } \\
\text { Observed } \\
\left(\mu \mathrm{g} \mathrm{m}^{-3}\right)\end{array}$} & $\begin{array}{c}\text { Mean } \\
\text { Predicted }\end{array}$ & NMB & NME & $\begin{array}{c}\text { Factor of } \\
2 \\
\end{array}$ \\
\hline & & & & $\left(\mu \mathrm{g} \mathrm{m}^{-3}\right)$ & $(\%)$ & $(\%)$ & $(\%)$ \\
\hline $\mathrm{CHO2}$ & Payerne & Switzerland & 2.54 & 2.02 & -21 & 70 & 48 \\
\hline DE44 & Melpitz & Germany & 2.52 & 4.25 & 69 & 85 & 66 \\
\hline ES1778 & Montseny & Spain & 4.52 & 6.35 & 40 & 88 & 67 \\
\hline IT04 & Ispra & Italy & 5.13 & 5.74 & 12 & 48 & 68 \\
\hline PL05 & $\begin{array}{c}\text { Diabla } \\
\text { Gora }\end{array}$ & Poland & 3.64 & 3.81 & 5 & 40 & 84 \\
\hline SI08 & Iskrba & Slovenia & 5.98 & 5.15 & -14 & 34 & 80 \\
\hline ALL & & & 4.06 & 4.55 & 15 & 61 & 69 \\
\hline
\end{tabular}

69

70

71

72

73

74

75 daily $\mathrm{PM}_{1} \mathrm{OA}$ measurements.

Table S7: Prediction skill metrics of PMCAMx-UF for the simulation without ELVOCs against daily $\mathrm{PM}_{2.5} \mathrm{OA}$ measurements. 
Table S8: Prediction skill metrics of PMCAMx-UF for the simulation without IVOCs against daily $\mathrm{PM}_{1}$ OA measurements.

\begin{tabular}{|c|c|c|c|c|c|}
\hline Station & $\begin{array}{c}\text { Mean } \\
\text { Predicted } \\
\left(\mu \mathrm{g} \mathrm{m}^{-3}\right)\end{array}$ & $\begin{array}{c}\text { Mean } \\
\text { Observed } \\
\left(\mu \mathrm{g} \mathrm{m}^{-3}\right)\end{array}$ & $\begin{array}{l}\text { NMB } \\
(\%)\end{array}$ & $\begin{array}{l}\text { NME } \\
(\%)\end{array}$ & $\begin{array}{c}\text { Factor of } 2 \\
(\%)\end{array}$ \\
\hline FIN & 2.44 & 2.12 & 15 & 26 & 93 \\
\hline PAT & 2.25 & 3.80 & -41 & 41 & 80 \\
\hline BOL & 3.88 & 5.68 & -32 & 36 & 78 \\
\hline SPC & 3.80 & 3.98 & -5 & 34 & 93 \\
\hline ALL & 3.12 & 3.79 & -18 & 35 & 87 \\
\hline
\end{tabular}

78

79

80

81

\begin{tabular}{|c|c|c|c|c|c|c|c|}
\hline \multirow{2}{*}{ Name } & \multirow[t]{2}{*}{ Station } & \multirow[t]{2}{*}{ Country } & \multirow{2}{*}{$\begin{array}{c}\text { Mean } \\
\text { Observed } \\
\left(\mu \mathrm{g} \mathrm{m}^{-3}\right)\end{array}$} & $\begin{array}{c}\text { Mean } \\
\text { Predicted }\end{array}$ & NMB & NME & $\begin{array}{c}\text { Factor of } \\
2 \\
\end{array}$ \\
\hline & & & & $\left(\mu \mathrm{g} \mathrm{m}^{-3}\right)$ & $(\%)$ & $(\%)$ & $(\%)$ \\
\hline $\mathrm{CHO2}$ & Payerne & Switzerland & 2.54 & 2.08 & -18 & 51 & 72 \\
\hline DE44 & Melpitz & Germany & 2.52 & 3.70 & 47 & 65 & 69 \\
\hline ES1778 & Montseny & Spain & 4.52 & 4.31 & -5 & 61 & 67 \\
\hline IT04 & Ispra & Italy & 5.13 & 3.50 & -32 & 45 & 65 \\
\hline PL05 & $\begin{array}{c}\text { Diabla } \\
\text { Gora }\end{array}$ & Poland & 3.64 & 3.56 & -2 & 38 & 87 \\
\hline SI08 & Iskrba & Slovenia & 5.98 & 3.78 & -37 & 37 & 80 \\
\hline ALL & & & 4.06 & 3.49 & -8 & 50 & 73 \\
\hline
\end{tabular}

82

83

84

85

86

87

88

89

Table S9: Prediction skill metrics of PMCAMx-UF for the simulation without IVOCs against daily $\mathrm{PM}_{2.5}$ OA measurements. 

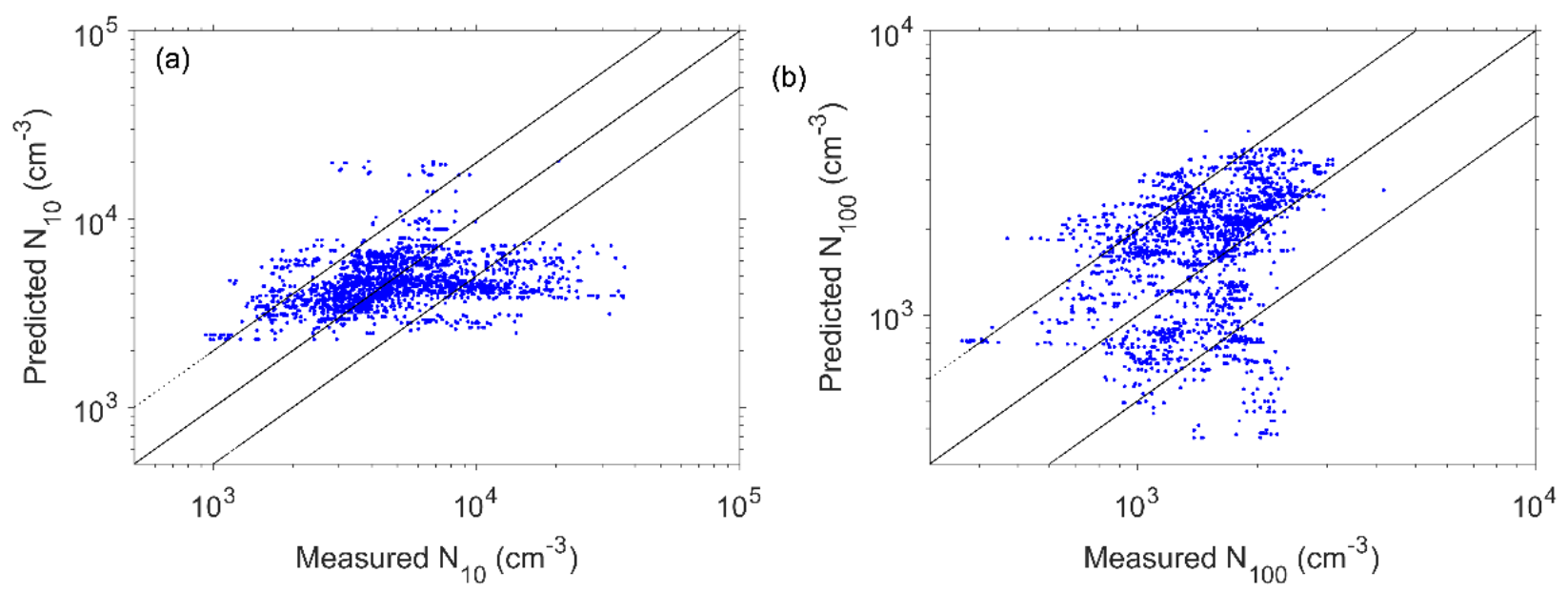

92 Figure S1: Comparison of predicted (base case) versus observed (Zeppelin) 3-min particle number 93 concentrations (in $\mathrm{cm}^{-3}$ ) for (a) $N_{10}$ and (b) $N_{100}$ of 25 flights over the Po Valley during the 94 PEGASOS campaign. There are approximately 2000 measurements included in the dataset. Also 95 shown the 1:1,2:1 and 1:2 lines.

96

97

98

99

100

101

102

103

104

105

106

107

108 

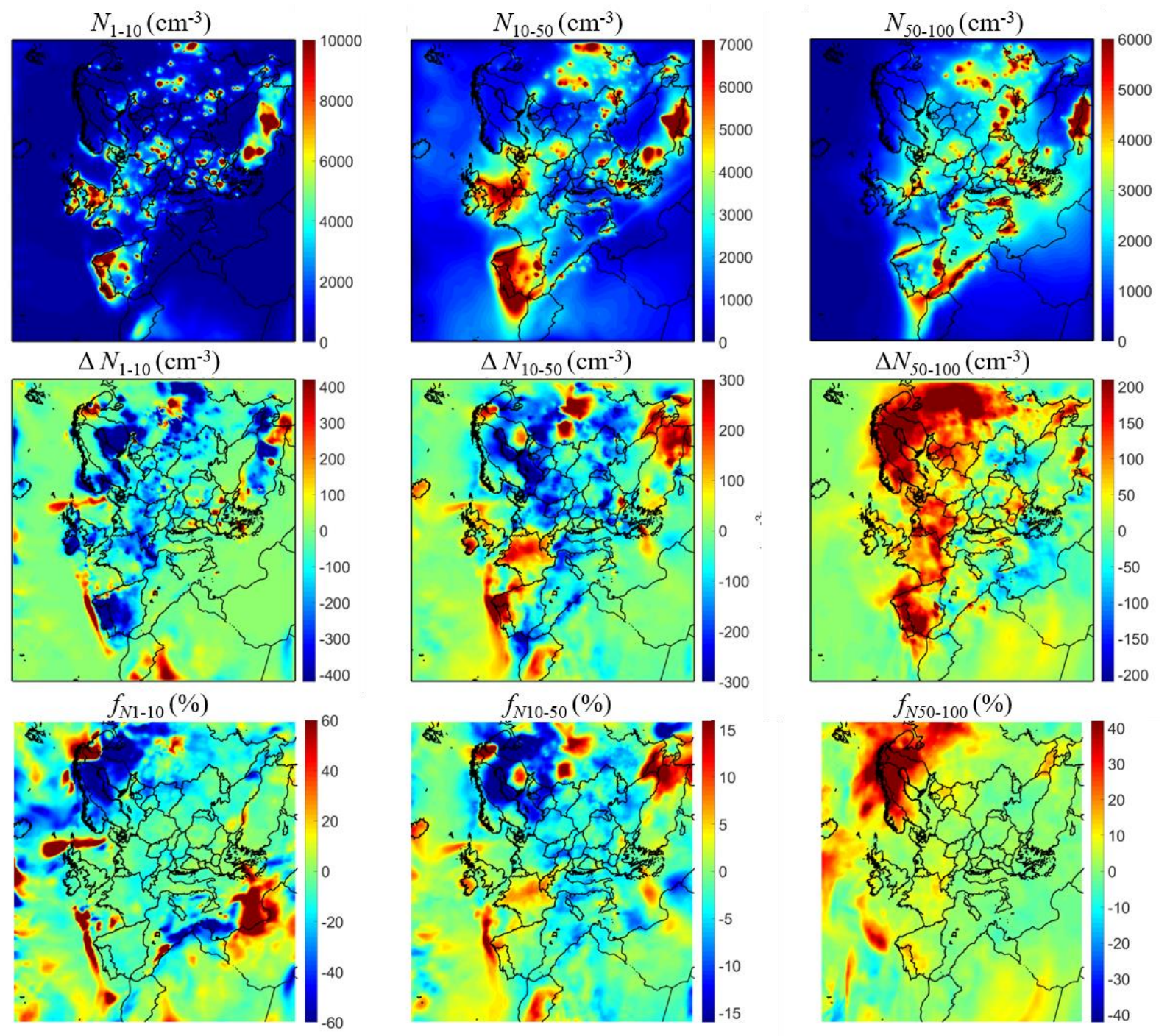

Figure S2: Average ground level number concentrations (in $\left.\mathrm{cm}^{-3}\right)(\mathrm{a}-\mathrm{b}-\mathrm{c})$, increase of number

112 the condensation of ELVOCs during 5 June - 8 July 2012 for: (a-d-g) particles between $0.8 \mathrm{~nm}$

113 and $10 \mathrm{~nm}\left(N_{1-10}\right)$; (b-e-h) particles between $10 \mathrm{~nm}$ and $50 \mathrm{~nm}\left(N_{10-50}\right)$ and (c-f-i) particles between

$11450 \mathrm{~nm}$ and $100 \mathrm{~nm}\left(N_{50-100}\right)$. Different scales are used. 

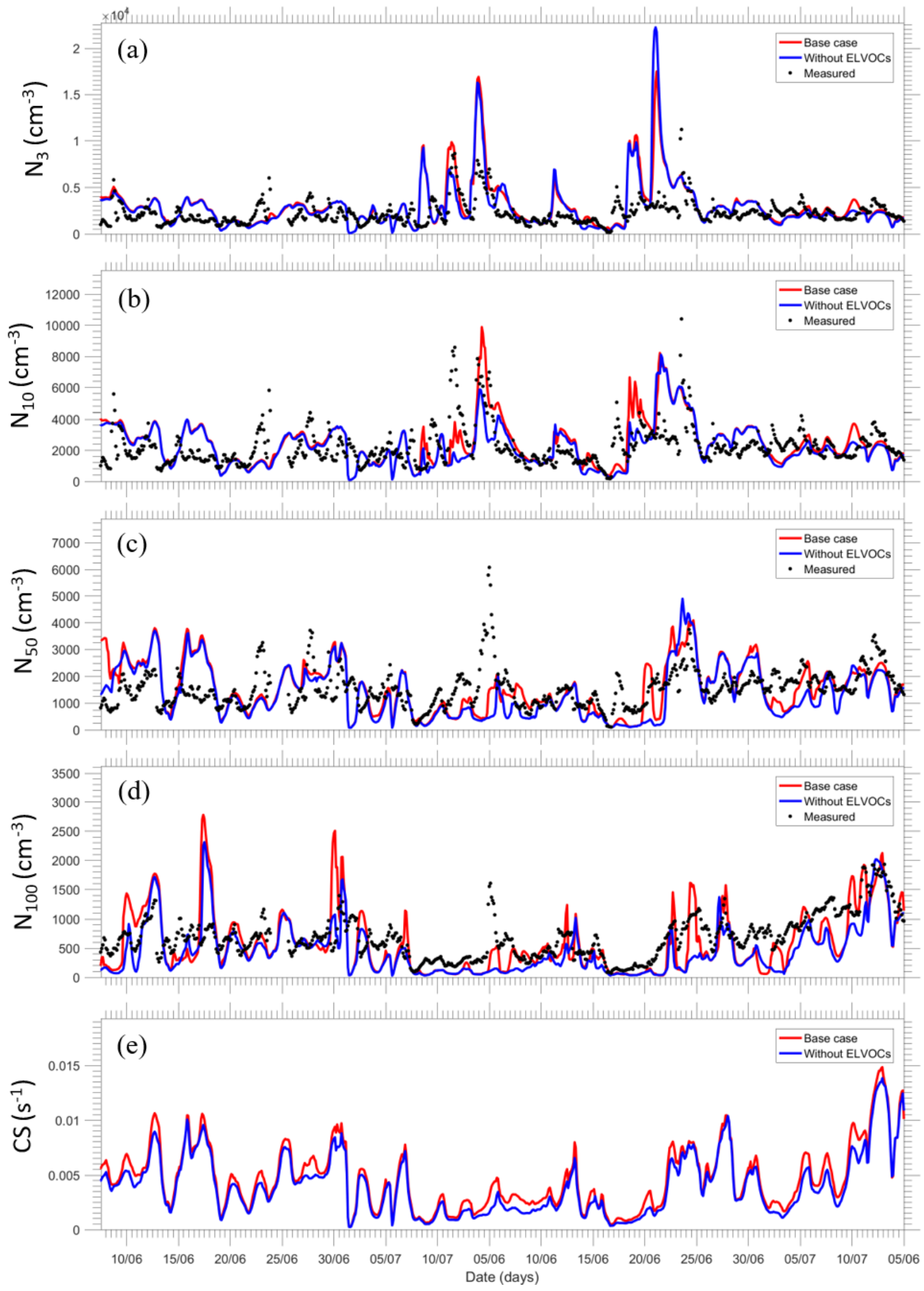

121 Figure S3: Time series of particle number concentrations (in $\mathrm{cm}^{-3}$ ) for (a) $N_{3}$, (b) $N_{10}$, (c) $N_{50}$, (d) $122 N_{100}$ and condensation sink (in $\mathrm{sec}^{-1}$ ) in Hyytiala site during 5 June -8 July 2012 with red line the 123 predictions of PMCAMx-UF for the base case, blue lines the prediction of PMCAMx-UF for the case without ELVOCs and the black dots are the measurements. 


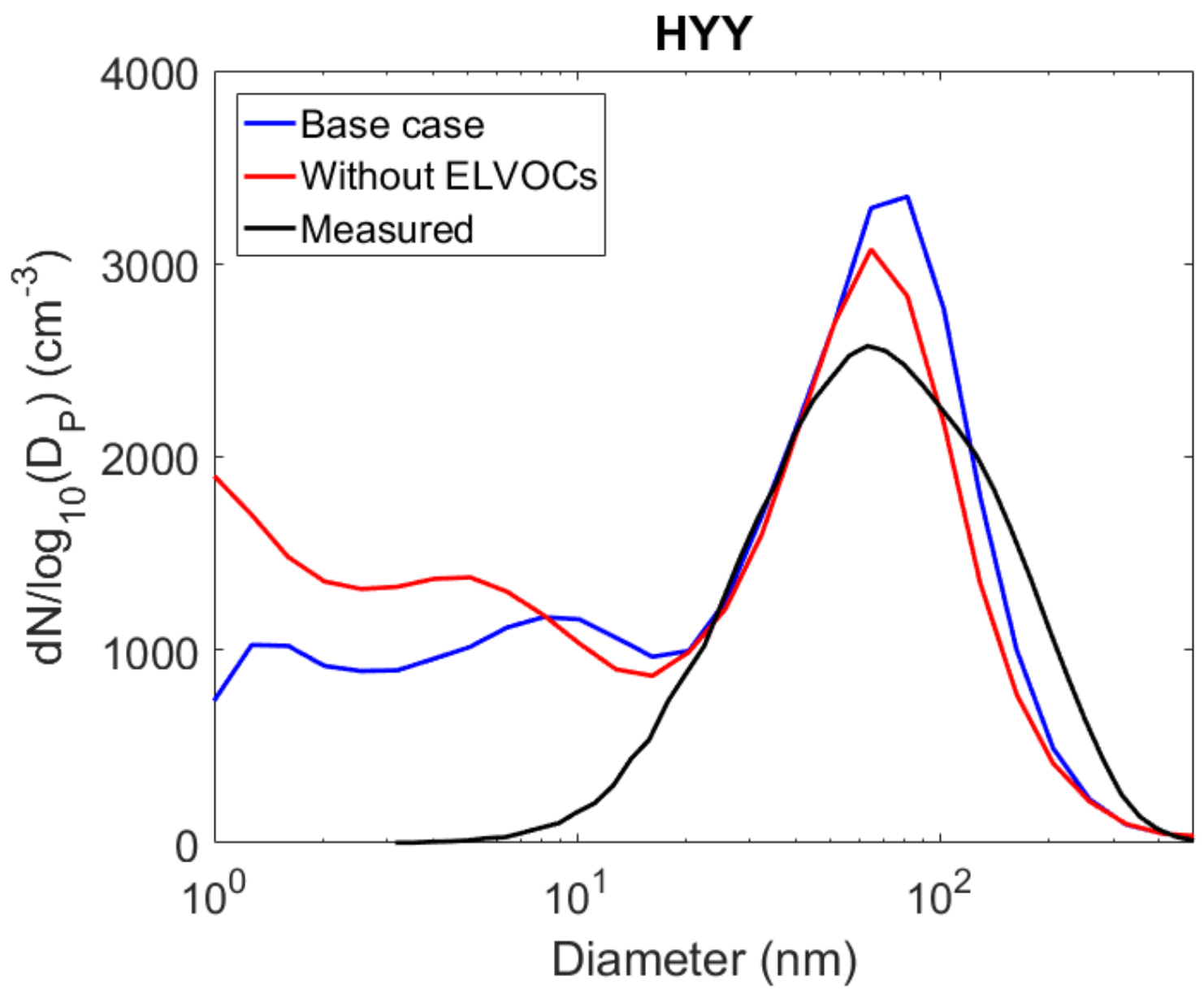

126 Figure S4: Average number size distribution in Hyytiala site during 5 June -8 July 2012. The 127 red line is the prediction of PMCAMx-UF for the base case simulation, the blue line is the 128 prediction of PMCAMx-UF for the case without ELVOCs and the black line is the measured 129 distribution (the smallest particles were not included in these measurements). 